
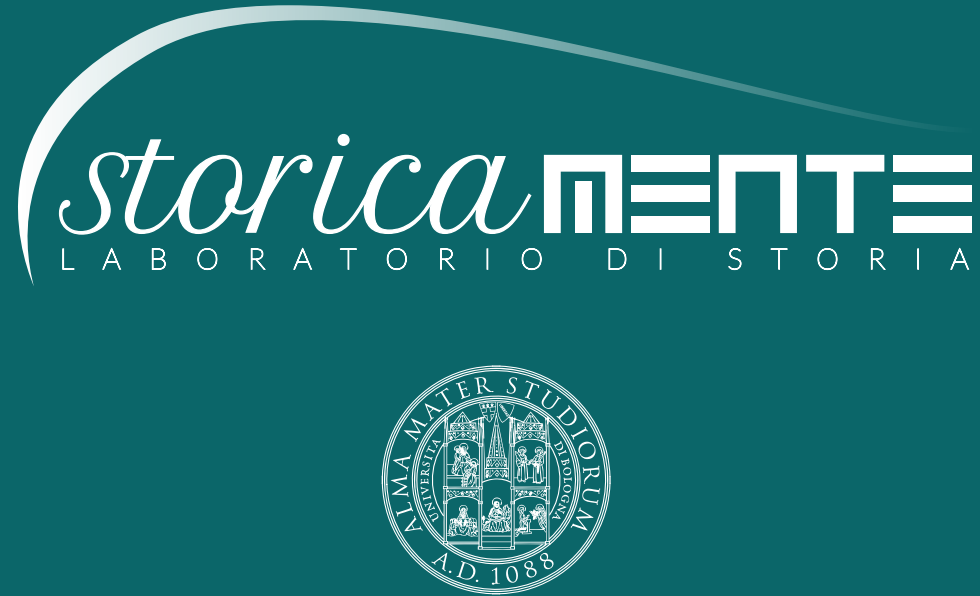

ALMA MATER STUDIORUM

Università di Bologna

Dipartimento di Storia Culture Civiltà

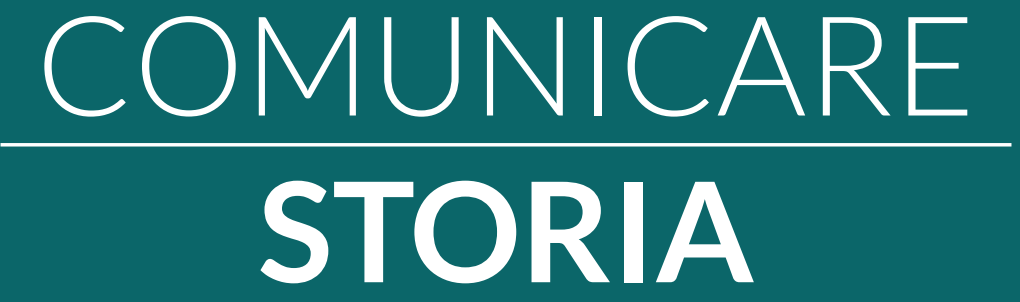




\section{STORICAMENTE.ORG}

\section{Laboratorio di Storia}

Alessio Fiore

Grandi narrazioni e lettura comparativa: metodo e prassi in "Framing the Early Middle Ages" di Chris Wickham

Numero $11-2015$

ISSN: $1825-411 \mathrm{X}$

Art. 11

pp. 1-13

DOI: $10.12977 /$ stor597

Editore: BraDypUS

Data di pubblicazione: 19/11/2015

Licenza: CC BY-NC-ND 4.0 International

Comunicare storia: Storiografie a confronto: linguaggi e concetti nelle traduzioni 


\title{
Grandi narrazioni e lettura comparativa: metodo e prassi in "Framing the Early Middle Ages" di Chris Wickham
}

\author{
ALESSIO FIORE \\ Dipartimento di Studi Storici \\ Università di Torinoi
}

Framing the Early Middle Ages di Chris Wickham, pubblicato nel 2005 e tradotto in italiano quattro anni più tardi, costituisce indubbiamente una delle opere più importanti pubblicate nel XXI secolo in campo storico [Wickham 2009a]. Si tratta di una imponente sintesi interpretativa incentrata, come è ben noto, sui mutamenti sociali e economici in Europa e nel bacino del Mediterraneo tra il 400 e l' 800 , che ha avuto (e sta ancora avendo) un fortissimo impatto in ambito storiografico. Questo perché non costituisce semplicemente una summa dei dati disponibili nel campo dell'alto medioevo al momento della stesura del testo, ma perché, grazie al suo taglio fortemente interpretativo, ha sollecitato la ricerca, talvolta spinta da una vis polemica, e quindi dalla volontà di falsificare o correggere alcune tesi o affermazioni dell'autore. Framing ha indubbiamente permesso un deciso salto qualitativo in avanti delle analisi, generando una maggiore consapevolezza di quelli che sono limiti dei singoli percorsi di ricerca e stimolando a superarli, con preziosi cortocircuiti.

Non mi occuperò qui delle tesi esposte, oggetto del resto di numerosi e approfonditi interventi critici e dibattiti, ma cercherò piuttosto di met- 
tere in luce alcuni degli aspetti di metodo a mio avviso più interessanti, connessi al tema più generale del seminario torinese, cioè quello della traduzione come strumento di comunicazione tra tradizioni storiografiche differenti. È forse non inutile sottolineare in questo senso che il mio rapporto con questo testo, e con i suoi problemi, nasce proprio dalla traduzione dello stesso in italiano, da me effettuata pochi anni fa con Luigi Provero. Tuttavia nelle prossime pagine non mi occuperò dei problemi tecnici da me affrontati in questa operazione, ma cercherò piuttosto di riflettere prima sull'importanza culturale della traduzione in italiano di un testo come Framing, per poi dedicarmi a esaminare alcune questioni metodologiche cruciali nel volume di Wickham che sono strettamente legate al problema della comunicazione tra le diverse tradizioni storiografiche nazionali.

\section{La traduzione nel contesto italiano}

La prima, e probabilmente banale, riflessione che vorrei provare ad articolare è relativa all'importanza della traduzione in italiano di un volume come Framing, da due diversi punti di vista, uno genericamente culturale, e uno più legato alla pratica della ricerca. Per il pubblico colto, ma non specialistico, l'alto medioevo è infatti ancora caratterizzato da letture di carattere ben diverso da quelle proposte da Wickham; la narrazione dominante è ancora quella della caduta dell'Impero, in un contesto di storia politico-militare, seppur arricchita negli ultimi anni da una crescente attenzione verso i complessi processi etnici e culturali a essa connessi.

Rispetto a questo approccio Framing incide in profondità su due diversi livelli: da un lato sostituisce l'idea di caduta con quella, più aggiornata, di trasformazione, e dall'altro propone una lettura che mette in modo molto deciso al centro del discorso l'economia, spesso considerata un fattore minore e tutto sommato separato rispetto alla struttura e all'as- 
setto dei poteri. Ed è proprio su questa centralità attribuita al dato economico che è a mio avviso particolarmente significativa la ricezione del volume di Wickham anche per la ricerca italiana; se infatti ci si sposta su un piano accademico, questo tipo di approccio rimane lontano anche dalla maggior parte della produzione scientifica italiana sull'alto medioevo, in cui risultano dominanti altre dimensioni: dai processi di costruzione etnica alle definizione dello status sociale, passando per la riflessione sulla produzione e fruizione delle fonti testuali [Gasparri, La Rocca 2013]. In un contesto di questo tipo anche i dati archeologici, del tutto centrali in Framing e sempre più largamente impiegati anche in Italia, vengono infatti usati generalmente ad altri fini, come lo studio dei lessici della superiorità sociale o la storia urbana. In questo contesto la traduzione di Framing potrebbe svolgere una funzione di indispensabile stimolo per riorientare proficuamente la ricerca italiana, offrendo nuovi approcci e prospettive, oggi ancora troppo trascurati, almeno rispetto alla storiografia in lingua inglese. Non è infatti casuale che negli stessi anni di Framing sia stato pubblicato in inglese un altro grande e importante volume, dello storico americano Michael McCormick, dedicato all'economia commerciale altomedievale. Niente di simile in Italia, ma le ricerche degli ultimi anni, in particolare quelle condotte dagli archeologi, ma non solo, mostrano una crescente attenzione verso la dimensione economica delle trasformazioni della società tra tardo antico e alto medioevo; un cambiamento di prospettiva, ancora non vistoso ma comunque percepibile, a cui l'ineludibile confronto con l'opera di Wickham non pare certo del tutto estraneo.

Tuttavia l'interesse di Framing non è semplicemente legato alla sua impostazione e alla proposta di rilettura complessiva offerta, e neppure allo straordinario esempio proposto di interazione tra fonti scritte e archeologiche. Il volume affronta infatti in modo deciso alcuni importanti nodi metodologici legati alla pratica stessa della ricerca storica: cruciali non solo per gli altomedievisti, ma per tutti coloro che di storia si occupano. Le scelte di Wickham in questo senso offrono decisivi spunti 
di riflessione e un modello empirico di tutto rilievo, come cercherò di mostrare nelle prossime sezioni.

\section{Grandi narrazioni}

In primo luogo per comprendere a fondo l'operazione compiuta da Wickham in Framing risulta fondamentale il concetto di "grande narrazione», che ritorna molto spesso nella prima parte del volume in cui ha un ruolo decisivo. Si tratta di un concetto introdotto dal filosofo francese Jean-François Lyotard, in La Condition postmoderne, un testo del 1979 incentrato proprio sull'analisi dei metarecits (un termine tradotto in italiano con grandi narrazioni, grand narratives in inglese) che strutturano il discorso [Lyotard 1979]. Con "grande narrazione» l'autore intendeva uno schema narrativo culturale totalizzante che ordina e spiega la conoscenza e l'esperienza; alcuni dei più significativi esempi di questo tipo erano costituiti dal "progresso», dalla «modernità», o ancora dall'«illuminismo». Questi metadiscorsi risultano tanto più condizionanti e strutturanti quanto più sono inconsci, impiegati cioè in modo irriflessivo; Lyotard adotta in questo senso una posizione di critica estrema di tali narrazioni e della loro efficacia, e sostiene che andrebbero eliminate per depurare il discorso, permettendo un approccio più libero e fecondo ai problemi.

Diverso sotto molti profili risulta l'approccio di Wickham, che in un importante articolo preparatorio a Framing pubblicato su «Storica» una decina di anni fa, ha affrontato di petto il tema delle grandi narrazioni in ambito storiografico, ritornando su questo argomento, in modo meno dettagliato, nell'introduzione al volume [Wickham 2003]. Sempre allo stesso tema è connesso l'intervento di Wickham alla "Settimana di Spoleto" dedicata al Feudalesimo, in cui rifletteva sui diversi significati di feudalesimo nelle varie tradizioni nazionali di studi [Wickham 2000]. Nello specifico storiografico per lo storico inglese le grandi narrazioni 
appaiono fortemente legate alle singole comunità accademiche nazionali; esse risultano connesse alla socializzazione accademica (e non solo accademica) dei membri delle diverse comunità scientifiche impegnate concretamente nella ricerca.

Anche dopo il superamento delle posizioni romantiche e nazionaliste tipiche di gran parte della storiografia ottocentesca l'alto medioevo è ancora oggi percepito, per motivi del tutto ovvi, come una fase critica per la costruzione delle singole entità nazionali e risulta quindi centrale nella costruzione della loro identità [Wood 2013]; con una felice espressione Wickham afferma che si tratta di una fase storica "viscerale» [Wickham 2003]. Il rilievo della fondazione delle singole comunità nazionali continua infatti ad avere una grande importanza per gli storici, più o meno consapevolmente. L'influenza delle grandi narrazioni determina il modo in cui ogni singola tradizione filtra i dati a sua disposizione, costruisce i propri questionari e genera scale di valore, in modo diverso a seconda dei casi. La crescente internazionalizzazione della ricerca in ambito medievistico, iniziata con la fondazione nel secondo dopoguerra del Centro Studi di Spoleto e che ha trovato negli ultimi anni un'accelerazione, anche grazie a progetti transnazionali come quello della "European Science Foundation" sulla Transformation of Roman World, ha solo parzialmente corretto questa situazione. Wickham afferma che «il problema di questi dibattiti è che, nelle loro forme nazionali, hanno pienamente senso solo per gli studiosi di una singola nazione, e talvolta non hanno alcun senso al di fuori dei suoi confini, se pure sono conosciuti dagli altri studiosi, ingabbiati all'interno delle proprie specifiche attenzioni nazionali» [Wickham 2009a, 30]. Una situazione che l'autore definisce poco più avanti una forma di «solipsismo culturale». La grande narrazione imperniata sulla città in Italia, quella sulla precocissima formazione dello stato-nazione in Inghilterra, quella sulla feodalité in Francia, risultano così profondamente radicate nelle rispettive tradizioni di studi da non potere essere pienamente comprese da chi proviene da contesti diversi. Solo il focalizzare l'attenzione 
sulle grandi narrazioni, prendendone pienamente consapevolezza, può quindi aiutare a disinnescarle, almeno parzialmente, riappropriandosi in una qualche misura di nuove angolazioni da cui osservare i dati a nostra disposizione.

In questo senso la formazione di Wickham e il suo personale percorso scientifico lo hanno messo in una posizione migliore rispetto ad altri per cogliere tali problemi. Dopo una formazione culturale e accademica in Inghilterra ha infatti studiato per molti anni l'Italia alto e pienomedievale (con ironia Wickham stesso parla, in un altro saggio, del suo stupore quando, ancora giovane studioso fresco di studi, venuto in Italia per le sue ricerche, si era accorto che anche gli italiani studiavano l'Italia altomedievale, cosa della quale, a Oxford, non si era affatto accorto). Proprio la sua natura di outsider, gli ha fornito l'opportunità di rendersi meglio conto di tali problemi; un'opportunità che, in modo non così scontato, ha colto perfettamente. Provenire da una tradizione accademica nazionale e dedicarsi allo studio di un altro paese spesso non costituisce infatti un antidoto efficace contro i problemi insiti nelle grandi narrazioni nazionali; almeno non per tutti. come infatti scrive lo stesso Wickham nell'introduzione a Framing, «non è stato pienamente efficace neppure un altro sviluppo che potenzialmente potrebbe dissolvere le tradizioni culturali nazionali dominanti in questo campo, ovvero l'esistenza di un ampio e crescente contingente di storici che studia un altro paese: sostanzialmente questi studiosi hanno la scelta tra farsi assorbire nei dibattiti nazionali dei paesi che studiano, oppure tenersene al di fuori, ma solo perché sono rimasti coinvolti nei dibattiti del proprio paese d'origine. Se fanno così possono aggiungere un elemento critico, e talvolta lo fanno, ma in questo caso sono spesso ignorati dagli storici del paese che studiano» [Wickham 2009a, 30-31].

Wickham, che in questo senso si distanzia nettamente da Lyotard, non vede le grandi narrazioni come un qualcosa di intrinsecamente falso e quindi da abolire per liberare il discorso. La grande narrazione imperniata sulla città in Italia ha senso perché effettivamente la città è molto 
importante in tale contesto. Occorre tuttavia acquisire consapevolezza delle loro origini ideologiche e cercare di inserire i dati a nostra disposizione in una cornice interpretativa rigorosa. Non si tratta quindi di abolire i quadri di riferimento, ma piuttosto di mirare a trasformarli, per quanto possibile, in veri e propri paradigmi scientifici (per usare la terminologia di Thomas Kuhn [1969]).

\section{Lettura comparativa e quadri concettuali}

Tale problema risulta particolarmente rilevante in Framing perché l'oggetto del libro sono ben 10 macro-regioni, corrispondenti in molti casi a uno o più degli attuali stati-nazione (dall'Italia, all'Egitto, dalla Spagna, alla Gallia franca); si tratta peraltro di regioni caratterizzate, oltre che da differenti grandi narrazioni nazionali, anche da sistemi di fonti molto diversi. La Danimarca, ad esempio si caratterizza per la presenza quasi esclusiva di fonti archeologiche, seppure molto abbondanti; per la Spagna sono invece disponibili fonti archeologiche, anche se non rilevantissime, e scritte, anche se queste ultime sono di carattere quasi esclusivamente normativo; Egitto e Irlanda presentano panorami ancora differenti. La sfida è quindi quella di mettere a confronto realtà così diverse, mettendo a punto strumenti analitici adatti ed efficaci. L'immagine delle matrice (insieme a quella del frame, della cornice) ricompare molto spesso nel corso del testo, e costituisce un forte antidoto proprio alle letture teleologiche insite nella ricerca su una specifica singola società. L'approccio comparativo consente infatti allo storico di avere a disposizione un meccanismo di controllo per comprendere i veri elementi causali dei mutamenti, scartando quelli empiricamente falsi [Wickham 2009b].

La diversità si spiega non attraverso la causa (come ad esempio la frattura causata dall'invasione islamica della penisola iberica) ma attraverso la costruzione di matrici che tengono conto di una pluralità di elementi 
che devono essere verificati in ogni singolo caso. Per esempio per spiegare il cambiamento socio-economico nel mondo post-romano, che costituisce il tema cardine del volume, è necessario tenere conto di una matrice di quattro elementi: la forza e la scala geografica del sistema fiscale in ogni regione e gli scambi economici che esso genera; la forza, scala geografica e ricchezza del possesso fondiario privato in connessione agli scambi economici cui dà vita; l'impatto della guerra e delle devastazioni belliche su ogni singola regione (molto pesante ad esempio in Italia o in Anatolia ma pressoché nullo in altre aree, come l'Egitto); e infine il grado in cui ogni regione era stata precedentemente integrata in modo strutturale nel sistema-mondo romano. Solo l'analisi empirica locale consente di vedere come tutti e quattro i parametri incidano su una data regione e come si intreccino, ma è solo la comparazione che ci consente di comprendere realmente la rilevanza di ogni singolo parametro evitando di fraintendere quelli che sono i nessi causali, attribuendo un'enfasi eccessiva a quello che in realtà è un dato non così decisivo (come l'invasione islamica della penisola iberica, ad esempio, a scapito di altri, meno appariscenti, ma di fatto più determinanti). Lo scopo di Wickham non è tanto, come già accennato in precedenza, quello di fornire una spiegazione, ma di costruire ed elaborare una cornice concettuale (un frame, di qui il titolo dell'opera) che ci consenta di formulare delle spiegazioni e di individuare dei nessi causali, di volta in volta differenti.

In questo contesto appare del tutto evidente che il problema centrale sia la necessità di tradurre (sia letteralmente, sia in senso lato) i risultati di tradizioni molto diverse tra loro in modo omogeneo per potere rendere possibile ed efficace il lavoro di comparazione. Le categorie analitiche e concettuali usate per filtrare i dati empirici diventano quindi cruciali. Ogni tradizione nazionale dispone infatti di un suo vocabolario, più o meno condiviso, più o meno soggetto a critiche e dibattiti (si pensi ad esempio al concetto di Stato altomedievale e al dibattito degli ultimi anni in Germania su questo tema, di cui tratta Giovanni Isabella nel suo 
contributo).

La scelta in questo ambito cruciale è molto netta. Wickham non si appoggia completamente ad alcuna tradizione nazionale (né a quella britannica né a quella italiana, solo per citare le due a cui l'autore per il suo personale percorso è più legato). C'è invece la creazione, attraverso quella che potremmo definire un'operazione di bricolage intellettuale - traendo cioè spunto da elementi presenti in tradizioni di studi diversi - di una serie di strumenti concettuali adatti a rendere conto dei dati empirici e ad attuarne la comparazione. I paradigmi sono infatti indispensabili allo storico, così come agli altri scienziati sociali. Si tratta di un'operazione concettuale che si sviluppa attraverso dei quadri analitici marxisti, come mostra l'importanza nel pensiero di Wickham del concetto di «modo di produzione». In questo senso è cruciale l'uso di idealtipi weberiani [Weber 2001]; lo storico britannico non prende a modello una realtà storica specifica, mettendola poi a confronto con le altre, ma costruisce, attraverso la giustapposizione di una serie di caratteristiche tra loro interrelate, un idealtipo, usandolo per ragionare sui casi concreti, riflettendo in particolare sui motivi per cui i vari elementi costitutivi funzionano di volta in volta in modo diverso, o risultano addirittura assenti.

Le etichette impiegate in tale procedimento risultano talvolta molto comuni; tra le più importanti nel libro si possono menzionare Stato, aristocratico, società feudale e società tribale. Tuttavia il contenuto di questi concetti, le realtà sociali, economiche e politiche che essi vanno a descrivere, sono almeno in parte differenti rispetto a quelli a cui il lettore, italiano, ma anche inglese, tedesco o spagnolo è abituato. Di qui anche l'estrema attenzione e cura con cui questi concetti o idealtipi vengono introdotti di volta in volta nel discorso. Non possono infatti venire dati per scontati, ma devono essere sempre, caso per caso, spiegati analiticamente in modo da consentire al lettore di avere chiaro quali sono gli attori sociali e politici o i processi economici a cui si fa riferimento quando si usano queste etichette. Ne illustrerò brevemente 
alcuni, gli stessi a cui ho fatto riferimento poco fa.

Il primo è quello dell'aristocratico. Wickham individua sei parametri principali per definire l'appartenenza di un individuo allo strato sociale aristocratico: questi sono l'ascendenza familiare, la ricchezza fondiaria, la collocazione in una gerarchia ufficiale, il favore imperiale o regio, il riconoscimento da parte degli altri protagonisti politici e lo stile di vita. Dalla combinazione di questi sei parametri (la cui specifica rilevanza locale è in assai variabile) emerge in modo chiaro quello che è l'idealtipo aristocratico all'interno del discorso portato avanti dall'autore [Wickham 2009a, 181-183].

Un secondo concetto chiave è lo Stato [Wickham 2009, 84-86]. Si intende qui per Stato un idealtipo caratterizzato da cinque criteri principali: la centralizzazione del legittimo uso della coercizione (giustizia ed esercito); la specializzazione nelle funzioni di governo, con la creazione di una gerarchia indipendente dagli individui che di volta in volta ne fanno parte; la nozione di potere pubblico, la presenza di risorse stabili e indipendenti a disposizione dei governanti e infine un sistema di classi per la stratificazione sociale e l'estrazione del surplus economico. Si tratta di un idealtipo che abbraccia quindi sia le robuste formazioni politiche del periodo romano e del Mediterraneo altomedievale (come il califfato e l'impero bizantino), sia quelle, decisamente più deboli dell'Occidente romano-germanico, ma non ad esempio, $\mathrm{i}$ regni irlandesi, anglossassoni o scandinavi, almeno per quanto riguarda il periodo fino all' 800 (con la parziale eccezione della Mercia).

Un terzo concetto chiave è quello di feudalesimo - e degli aggettivi derivati - [Wickham 2009a, 85-88], un lemma assai problematico caratterizzato com'è da fortissime differenze semantiche nelle diverse tradizioni storiografiche [Wickham 2000]. Qui è usato per indicare una società in cui esiste il concetto di proprietà della terra, in cui i signori fondiari dominano sui contadini e vivono grazie al surplus prodotto dagli affittuari che lavorano le terre di loro proprietà. Si tratta di un'accezione molto prossima a quella tipica della storiografia spagnola, ma 
assai lontana ad esempio da quello tipico dell'Italia, in cui il termine è usato in modo più tecnico per indicare le relazioni vassallatico-beneficiarie, ma anche da quello comune in Francia [Barbero-Vigil 1979]. L'ultimo concetto che che vorrei presentare è quello di società tribale [Wickham 2009a, 335-336]. Esso indica in Framing una società in cui gran parte dei contadini sono produttori indipendenti, e in cui i potenti, che pure sono presenti, dominano di fatto solo su un settore limitato della società contadina, in cui i contadini nel loro complesso hanno obblighi (tributi e prestazioni militari) nei confronti di chi comanda, ma sono largamente autonomi sotto il profilo economico; inoltre queste società sono spesso caratterizzate addirittura dall'assenza del concetto di proprietà fondiaria esclusiva. Questo concetto, quello di società tribale, è impiegato non solo per descrivere realtà sociali come quella della Britannia anglosassone prima del VII secolo, o della Mauritania berbera, senza suscitare particolari sorprese, ma anche quelle degli Appennini o del Chianti meridionale altomedievali; e in quest'accezione risulta, come è evidente al lettore, del tutto assente dalla tradizione di studi italiana che di questi contesti sociali e storici si è occupata.

Anche se ho presentato questi concetti in modo molto più schematico rispetto a quanto non avvenga nel volume, risulta comunque del tutto evidente la precisione con cui essi sono introdotti nel discorso e articolati, per poi successivamente essere impiegati nell'analisi. Mi preme sottolineare inoltre il fatto che questi concetti associati ai singoli idealtipi o concetti sono da considerare per Wickham strettamente legati all'operazione analitica condotta in Framing. In altri contesti appaiono invece del tutto legittimi altri significati (anche se ciò non significa certo che tutti i significati risultano legittimi). Ad esempio lo stesso autore ha fatto uso del termine feudalesimo con accezioni molto differenti tra loro in testi diversi, rivendicando proprio tale libertà di uso, a patto che di volta in volta si precisi il senso da attribuire all'etichetta per evitare equivoci e fraintendimenti da parte del lettore.

L'operazione intellettuale di Framing risulta dunque estremamente utile 
non solo da un punto di vista empirico e interpretativo, ma anche, più in generale, da quello strettamente metodologico. Due sono i punti cruciali; da un lato si mostra, ancora una volta, l'importanza e le potenzialità euristiche della strategia comparativa (come pure il rigore con cui essa deve essere attuata), oggetto del resto negli ultimi anni di un rinnovato interesse e di sempre più serrate riflessioni metodologiche [Diamond-Robinson 2011]. Forse meno evidente, ma secondo me cruciale, è il secondo messaggio: quello della necessità di riflettere sul contenuto della "cassetta degli attrezzi" dello storico, di non dare cioè per scontati gli strumenti che ci sono all'interno, ma di usarli in modo estremamente consapevole ed esplicito. Impiegarli inconsapevolmente, dando per scontato il loro senso e significato, fa infatti sì che siano gli strumenti e i concetti a usare noi e non viceversa.

\section{Bibliografia orientativa}

Barbero A., Vigil Pascual M. 1978, La formación del feudalismo en la Península Ibérica, Barcelona: Editorial Crítica.

Diamond J., Kirch P. V., Robinson J. A. 2011, Esperimenti naturali di storia, Torino: Codice.

Gasparri S., La Rocca C. 2012, Tempi barbarici: l'Europa occidentale tra antichità e Medioevo (300-900), Roma: Carocci.

Haldon J. F. 1993, The state and the tributary mode of production, London; New York: Verso.

Kuhn T. S. 1969, La struttura delle rivoluzioni scientifiche, Torino: Einaudi (ed. or. 1962).

Lyotard J. F. 1979, La condition postmoderne: rapport sur le savoir, Paris: Les Éditions de Minuit.

Mann M. 1986, The sources of social power. Vol. 1, Cambridge: Cambridge University Press.

McCormick M., Sampaolo M. 2008, Le origini dell'economia europea: comunicazioni e commercio 300-900 d. C., Milano: V\&P (ed. or. 2002).

Runciman W. G. 1989, A treatise on social theory, Vol. 2, Cambridge [Cambridgeshire]; New York: Cambridge University Press. 
Weber M. 2001, L'oggettività conoscitiva della scienza sociale e della politica sociale, in Id., Saggi sul metodo delle scienze storico-sociali, Torino: Edizioni di Comunità, 187-191.

Wickham C. 2003, Alto medioevo e identità nazionale, «Storica», IX (27): 7-26.

- 2000, Le forme del feudalesimo, in Il Feudalesimo nell'Alto Medioevo, I, (Settimane del Centro Italiano di Studi sull'Alto Medioevo XX), Spoleto: CISAM, 15-46.

- 2009a, Le società dell'alto medioevo. Europa e Mediterraneo, secoli V-VIII, La storia. Saggi, Vol.2, Roma: Viella (ed. or. Framing the Early Middle Ages. Europe and the Mediterranean 400-800, Oxford 2005).

- 2009b, Problems in Doing Comparative History, in Skinner P. (ed.), Challenging the boundaries of medieval history, Turnhout: Brepols, 5-28.

Wood I. N. 2013, The modern origins of the early Middle Ages, Oxford: Oxford University Press. 